\title{
Fast Auroral Imager (FAI) for the e-POP Mission
}

\author{
Leroy Cogger • Andrew Howarth • Andrew Yau • Andrew White • Greg Enno • \\ Trond Trondsen • Don Asquin • Blair Gordon • Paul Marchand · Danny Ng • \\ Greg Burley • Marc Lessard • Brent Sadler
}

Received: 30 May 2014 / Accepted: 27 September 2014 / Published online: 22 October 2014

(C) The Author(s) 2014. This article is published with open access at Springerlink.com

\begin{abstract}
The Fast Auroral Imager (FAI) consists of two charge-coupled device (CCD) cameras: one to measure the $630 \mathrm{~nm}$ emission of atomic oxygen in aurora and enhanced night airglow; and the other to observe the prompt auroral emissions in the 650 to $1100 \mathrm{~nm}$ range. High sensitivity is realized through the combination of fast lens systems $(f / 0.8)$ and CCDs of high quantum efficiency ( $>90 \%$ max). The cameras have a common 26 degree field-of-view to provide nighttime images of about $650 \mathrm{~km}$ diameter from apogee at $1500 \mathrm{~km}$. The near infrared camera provides up to two images of $0.1 \mathrm{~s}$ exposure per second with a spatial resolution of a few $\mathrm{km}$ when the camera is pointing in the nadir direction, making it suitable for studies of dynamic auroral phenomena. The 630-nm camera has been designed to provide one image of $0.5 \mathrm{~s}$ exposure every 30 seconds. Launch of the satellite occurred on September 29, 2013. Following a description of the instrument, sample auroral images are presented.
\end{abstract}

Keywords Satellite $\cdot$ Imager $\cdot$ Aurora $\cdot$ Ionosphere $\cdot$ Magnetosphere

L. Cogger $(\varangle) \cdot$ A. Howarth · A. Yau $\cdot$ A. White $\cdot$ G. Enno

Dept. of Physics and Astronomy, University of Calgary, Calgary, Alberta, Canada

e-mail: lcogger@phys.ucalgary.ca

T. Trondsen

Keo Scientific, Calgary, Alberta, Canada

D. Asquin

Magellan Aerospace, Ottawa, Ontario, Canada

B. Gordon · P. Marchand · D. Ng

Routes Astroengineering (formerly), Kanata, Ontario, Canada

G. Burley

Burley Scientific, Vancouver, BC, Canada

M. Lessard · B. Sadler

Space Science Center, University of New Hampshire, Durham, NH, USA 


\section{Introduction}

The Fast Auroral Imager (FAI) is part of the Enhanced Polar Outflow Probe (e-POP) instrument suite on the Canadian Cassiope small satellite, which was launched by Space Exploration Technologies Corporation (SpaceX) from Vandenberg Air Force Base on September 29,2013 into a 325 by $1500 \mathrm{~km}$ elliptical orbit at $81^{\circ}$ inclination. The major scientific objectives of the e-POP mission are associated with the Earth's high latitude atmosphere and ionosphere. The first thrust is to investigate the outflow of plasma from the polar regions and the related processes of microscale ion acceleration and wave-particle interaction, and auroral excitation. The second thrust is the study of $3 \mathrm{D}$ ionospheric irregularities using both active and passive radio techniques. The third emphasis is on the escape of neutral particles from high latitudes caused by temperature enhancements as well as by non-thermal processes. The mission concept therefore naturally requires the highest possible in-situ measurements of electrons and ions, techniques for 3D radio wave propagation studies, and high temporal and spatial resolution images of mesoscale optical aurora from space. For a comprehensive overview of the mission, see Yau et al. (2006).

The Fast Auroral Imager was developed to meet the optical imaging objectives of the mission. Canadian scientists and their industrial partners have a long history of auroral imaging, beginning with the ISIS2 satellite (Anger et al. 1973) that was launched in 1971 to investigate visible aurora during nighttime hours. For the next two decades, imagers were developed and flown for the purpose of studying the global distribution of FUV aurora under both sunlit and dark atmospheric conditions. These imagers used intensified charge-coupled device (CCD) detectors to achieve the necessary sensitivity as part of the Swedish Viking and Freja missions, and the Russian Interball mission (Anger et al. 1987; Murphree et al. 1994; Cogger et al. 2002). In this paper we describe the latest stage in Canadian imager development: the application of CCD technology to achieve a combination of high spatial and temporal resolution with high sensitivity using optical (visible and near infrared) aurora as the target. These emissions are visible at night both from the ground and from space. In the U.S.A., interest has been mostly directed to imaging the entire auroral oval from high altitudes as part of large missions, e.g. DE (Frank and Craven 1981), POLAR (Germany et al. 1998), IMAGE (Burch 2000).

\section{Science Objectives and Measurement Requirements}

In keeping with the overall scientific objectives for the e-POP mission, a list of objectives were identified for the auroral imager to guide the measurement requirements and the instrument design. The general aim is to investigate the relationship between auroral emissions and ion bulk upflow, heating, acceleration, field-aligned currents, and plasma waves. In more specific terms, the FAI was designed to support the investigation of the following: (a) the small-scale mechanisms for auroral excitation, (b) the phenomenon of black aurora and its relation to streaming electrons and ions, (c) the optical signatures of large-scale convection and their relation to radar measurements, and (d) the connections between aurora and ion energization and bulk upflow.

The above goals are related to a wide range of auroral intensity and temporal/spatial scales. To accommodate this range, it is necessary to observe aurora in the visible and nearinfrared portions of the spectrum rather than the FUV. The spatial resolution must be selectable, with a minimum pixel size corresponding to several $\mathrm{km}$ at auroral altitudes when observed from apogee. The temporal resolution and exposure time must also be selectable, 
with temporal resolution as small as a fraction of one second. A repetition rate of better than one second is achievable with a 3-axis stabilized platform and fast data transfer. Because the spatial and temporal characteristics of auroras generated by low and high energy electrons can be very different, it is desirable to incorporate the capability to measure both independently by the use of two cameras whose parameters are chosen for this purpose. Unlike many imaging missions, e-POP does not have a requirement for global imaging because the target is mesoscale aurora that is of the order of a few hundred kilometers rather than thousands of kilometers. The FAI was designed for nightside operation in the visible and near infrared spectral regions, and therefore refractive rather than reflective optics could be utilized to reduce the cost.

\section{Instrument Concept}

The measurement requirements presented a challenge to design an auroral imaging instrument that would make advances in both temporal and spatial resolution as well as sensitivity and dynamic range. The spatial resolution capability for most auroral imagers over the past few decades has been of the order of $10 \mathrm{~s}$ of $\mathrm{km}$ whereas the need in the e-POP mission was for an order of magnitude improvement. Likewise, a similar improvement in repetition rate was required. The challenge was met by carefully selecting the spectral elements to image and by taking advantage of the best available technology within the constraint of a very limited budget. It was decided to design two cameras that are co-aligned and identical in all respects except for the optical filters. One camera (VIS) images the emission from the atomic oxygen line at $630.0 \mathrm{~nm}$. This emission is pervasive throughout the auroral region and polar cap, and it is excited efficiently by low-energy electrons. It is very useful for identifying features and boundaries in the atmosphere that correspond to different processes in the magnetosphere. However, because of the relatively long radiative lifetime, it is not a suitable emission to utilize for high temporal and spatial resolution so the second camera (NIR) was designed to image a range of auroral emissions in the 650 to $1100 \mathrm{~nm}$ part of the spectrum. The principal emission species in this range, $\mathrm{N}_{2}, \mathrm{O}_{2}$ and $\mathrm{N}_{2}^{+}$, respond preferentially to electrons with energies higher than those for the VIS camera and closely follow the dynamic variations of the precipitating particles.

The sensitivity of the cameras was maximized further through the choice of a CCD detector and optics module. We chose a thinned, backside-illuminated, high quantum efficiency and low noise CCD, the e2v CCD67 in a 256 by 256 pixel array. According to the manufacturer, the quantum efficiency is 0.8 at $630 \mathrm{~nm}$, and effectively about 0.66 for the NIR camera, and the read noise is less than 10 electrons. With thermal-electric cooling of the advanced inverted mode operation (AIMO) back illuminated device, the dark current can be kept insignificant for normal instrument operating temperatures. As for the optics, we combined an $f / 4$ telecentric lens system with a 5:1 fibre-optic taper to provide an effective f-number of 0.8 . The price paid for the use of the taper is a reduction in optical transmission.

The electronic control system was designed to permit a selection of exposure times and on-chip summation in order to adjust to the various science modes during the mission. A large dynamic range was preserved through the use of 16-bit data words and a 16-bit analog-to-digital converter.

\section{Instrument Design}

The design and construction tasks were distributed to several companies. The optics modules were designed and built by Coastal Optical Systems under the supervision of Keo Con- 
sultants. Burley Scientific was responsible for the control and readout electronics as well as the flight software. Routes AstroEngineering (now COM DEV) undertook the mechanical design and construction of the camera bodies, the electronics housing, the baffles, the mounting of the CCDs and the thermoelectric cooling of the detectors. Routes also had the overall responsibility for the assembly of the cameras. Table 1 summarizes the instrument design parameters.

\subsection{Mechanical Overview}

The FAI is composed of two mechanical structures. One structure contains both cameras and the other contains the electronics. A cut-away drawing of the camera unit is shown in Fig. 1. The baffles are designed for a field of view of $27.5^{\circ}$, which is slightly larger than the optical field of view to keep the vanes out of the camera field of view. Four knife-edge vanes are positioned to maximize the effectiveness of the baffles whose length is constrained by the spacecraft mounting requirements. The optics modules are rigidly attached to the camera unit, and the fibre optics block supporting each CCD is bonded to the small end of the fibre optic 5:1 taper. The electronics box is independently mounted to the deck plate of the satellite. The camera unit and electronics box have a combined mass of $6.72 \mathrm{~kg}$.

\subsection{Optical System Overview}

There are seven anti-reflection coated lenses in the optical system that produce an image diameter of $33 \mathrm{~mm}$ on the input end of the fibre optic taper. A circular image of diameter $6.6 \mathrm{~mm}$ is formed on the $6.6 \mathrm{~mm}$ by $6.6 \mathrm{~mm} \mathrm{CCD}$. The full-angle field of view of each $f / 4$ camera is $26^{\circ}$. The design is telecentric to ensure that the same cone angles are seen by all parts of the filters. The focal length of the system is $68.9 \mathrm{~mm}$. The VIS camera contains a metal oxide interference filter of $2 \mathrm{~nm}$ half-width and peak transmission of 0.8 manufactured by Barr Associates. The metal oxide design minimizes passband shifts from $630.3 \mathrm{~nm}$ due to temperature variations. The filter in the NIR camera is a broadband filter, Schott RG645, that has a transmission of 0.9 over the range 650 to $1100 \mathrm{~nm}$. Shorter wavelengths are blocked. The lenses are supported in a titanium housing.

The CCDs are cooled by means of two-stage thermal-electric coolers (TEC) that produce a maximum temperature difference of about $-25^{\circ} \mathrm{C}$ relative to the instrument's baseplate temperature. Optimum performance is achieved with a baseplate temperature of $15{ }^{\circ} \mathrm{C}$ or lower, resulting in a CCD temperature of $-10{ }^{\circ} \mathrm{C}$ or lower. At these low temperatures the rate of generation of thermal electrons within the $\mathrm{CCD}$ is lower than 10 electrons/s. During operation in orbit, the FAI baseplate temperature is typically close to $-5{ }^{\circ} \mathrm{C}$ allowing the CCDs to be routinely cooled to $-20{ }^{\circ} \mathrm{C}$.

\subsection{Electronic System Overview}

Figure 2 is a block diagram showing the various parts of the sensor system. The controller provides bias voltages, three phase clock signals to the CCD parallel and serial registers, signal processing to extract and digitize the CCD output signals, and a fast serial interface to a data handling unit (DHU) on the spacecraft. The electronic design is similar to that of the Microvariability and Oscillations of Stars Telescope (MOST) (Walker et al. 2003) that has been operating for more than 10 years.

The digital signal processor (DSP) directly generates the sequences used to clock the serial and parallel charge transfers on each CCD. Extra clock lines are provided for frame transfer. The DSP also controls the dual-slope integrator, the analog-to-digital (A/D) 
Table 1 FAI instrument parameter data table

\begin{tabular}{|c|c|c|c|c|}
\hline Category & Item & Parameter & Value & Source \\
\hline Optics & Baffle & FOV & $27.5^{\circ}$ & Routes Astroengineering \\
\hline Optics & Baffle & Vanes & 4 & Routes Astroengineering \\
\hline Optics & Lenses & Elements & 7 & Coastal Optical Design \\
\hline Optics & Lenses & Focal Length & $68.9 \mathrm{~mm}$ & Coastal Optical Design \\
\hline Optics & Lenses & f-number & 4.0 & Coastal Optical Design \\
\hline Optics & Lenses & Field of view & $26^{\circ}$ (full) & Coastal Optical Design \\
\hline Optics & Lenses & Image Size & $33 \mathrm{~mm}$ diam. & Coastal Optical Design \\
\hline Optics & Lenses & Transmission & 0.8 & Estimate (18 surfaces) \\
\hline Optics & NIR filter & Transmission & $0.9(\lambda>650 \mathrm{~nm})$ & Schott RG645 $3 \mathrm{~mm}$ \\
\hline Optics & VIS filter & Transmission & $0.68(\lambda=630.3 \mathrm{~nm})$ & $\operatorname{Barr}\left(14^{\circ}\right.$ cone $)$ \\
\hline Optics & VIS filter & Half-width & $2.0 \mathrm{~nm}$ & $\operatorname{Barr}\left(14^{\circ}\right.$ cone $)$ \\
\hline Optics & Taper & Reduction & $5: 1$ & Schott Fiber Optics \\
\hline Optics & Taper & Image circle & $6.6 \mathrm{~mm}$ diam. & Coastal Optical Design \\
\hline Optics & Taper & Transmission & 0.5 & Estimated \\
\hline CCD & & Pixel size & $26 \mu \mathrm{m} \times 26 \mu \mathrm{m}$ & e2v CCD67 \\
\hline $\mathrm{CCD}$ & Quan. Eff. & VIS band & 0.92 & e2v CCD67 \\
\hline $\mathrm{CCD}$ & Quan. Eff. & NIR band & 0.66 effective & Calculated \\
\hline CCD NIR & & Dark current & $529 \mathrm{e}^{-/ \mathrm{s}} @ 293 \mathrm{~K}$ & e2v spec sheet \\
\hline CCD VIS & & Dark current & 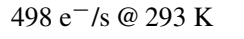 & e2v spec sheet \\
\hline $\mathrm{CCD}$ & & Full well & $600 \mathrm{~K}$ electrons & e2v spec sheet \\
\hline CCD & & Readout & 0.33 seconds & Burley Scientific \\
\hline CCD & & Read noise & 10 electrons rms & Measured \\
\hline $\mathrm{CCD}$ & & Output & $1.5 \mu \mathrm{V} / \mathrm{e}^{-}$ & e2v CCD67 \\
\hline Electronics & $\mathrm{ADC}$ & Input & 0 to $5.0 \mathrm{~V}$ & Burley Scientific \\
\hline Electronics & $\mathrm{ADC}$ & Output & 16 bits & Burley Scientific \\
\hline Electronics & $\mathrm{ADC}$ & Conv. Time & $1.25 \mu \mathrm{s} /$ pixel & Burley Scientific \\
\hline Electronics & Conversion & VIS & $1.4 \mathrm{e}^{-} / \mathrm{DN}$ & Measured \\
\hline Electronics & Conversion & NIR & $1.4 \mathrm{e}^{-} / \mathrm{DN}$ & Measured \\
\hline General & & Power & $7-17.9 \mathrm{~W}$ & Measured \\
\hline General & & Mass & $6.72 \mathrm{~kg}$ & Measured \\
\hline General & & Telemetry & $1.5 \mathrm{Mbits} / \mathrm{s}$ & FAI ICD \\
\hline Default & Binning & VIS & 2 by 2 & \\
\hline Default & Binning & NIR & 1 by 1 & \\
\hline Default & Exposure & VIS & $0.5 \mathrm{~s}$ & \\
\hline Default & Exposure & NIR & $0.1 \mathrm{~s}$ & \\
\hline Default & Cycle time & VIS & $30 \mathrm{~s}$ & \\
\hline Default & Cycle time & NIR & $1.0 \mathrm{~s}$ & \\
\hline Default & Responsivity & VIS & $0.127 \mathrm{DN} / \mathrm{R} \cdot \mathrm{s}$ & Measured \\
\hline Default & Responsivity & NIR & $0.0965 \mathrm{DN} / \mathrm{R} \cdot \mathrm{s}$ & Measured \\
\hline Orbit & Period & & $103 \mathrm{~min}$ & Measured \\
\hline Orbit & Apogee & & $1500 \mathrm{~km}$ & Measured \\
\hline Orbit & Perigee & & $325 \mathrm{~km}$ & Measured \\
\hline Orbit & Inclination & & $81^{\circ}$ & Measured \\
\hline
\end{tabular}


Fig. 1 Cutaway drawing of the camera unit

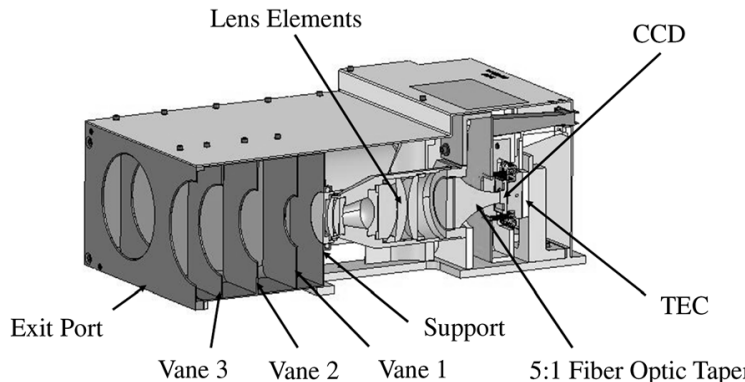

Vane 3 Vane 2 Vane 1 5:1 Fiber Optic Taper

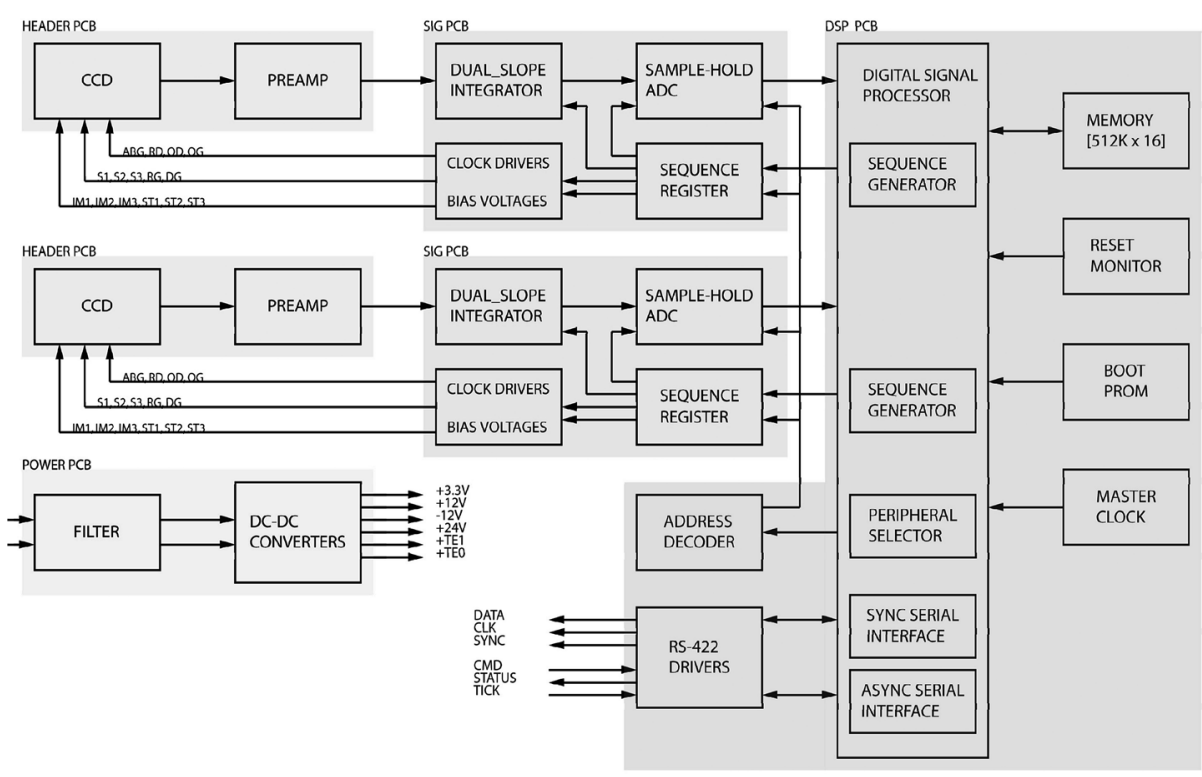

Fig. 2 Block diagram of the FAI electronics

conversion, and the data transfer to the interface. The design of the dual-slope integrator and clock drivers are optimized for fast settling time.

The design is partitioned into three parts. Each CCD is packaged with a low-noise preamplifier and a temperature sensor. A cable connects each CCD to the main electronics unit, which contains all the support circuitry to operate the CCDs and communicate with the DHU. The CCD is a frame-transfer device; therefore no mechanical shutter is required.

\subsection{Software Description}

Development of the software used the TASKING DSP C language cross-compiler and development tools (v3.0 or higher). The DSP code is written in a combination of C and assembly language to run on the DSP56309 processor in the FAI controller. The flight software for FAI has been adapted from existing software for the MOST camera, and has a similar structure.

The code is built in three sections: (1) a set of initialization routines, (2) an event loop that decodes the commands sent to the camera over the serial link, and (3) a set of subroutines to implement the decoded commands. 


\section{Instrument Operations}

\subsection{Operating Modes}

The operating modes (per camera) for FAI are listed in Table 2.

Each operating mode is defined by a set of parameters, including the image size, binning, nominal exposure time and cycle time. These values are stored in an "uploadable" table in the DSP memory.

The parameter table is an area allocated in memory to store the operating parameters of the two CCDs. The parameter table includes one operating mode table per CCD. Values in the parameter table are accessible using an offset number as an index into the table.

Independent parameters include the CCD temperature set point and/or the TEC current set point (for open loop operation) for each CCD.

In operation, the DSP copies the values for the selected mode(s) from the table to an active buffer. The value of each active parameter can be changed individually. For example, the high-res mode might be selected for FAI-NIR and the exposure time changed to $120 \mathrm{~ms}$ without having to change the complete table.

The default operating parameters are given in Table 3 for each camera. Calculations of projected pixel size are shown for a satellite orbit apogee of $1500 \mathrm{~km}$ and perigee of $325 \mathrm{~km}$. The assumed altitude of the aurora is $220 \mathrm{~km}$ for the $630 \mathrm{~nm}$ and $110 \mathrm{~km}$ for the near infrared emissions. A single pixel of size of 26 by 26 microns limits the optical resolution of the

Table 2 FAI operating modes

\begin{tabular}{ll}
\hline Mode & Description \\
\hline IDLE/HK & $\begin{array}{l}\text { Powered on, TEC off, no data transmitted, operating in } \\
\text { housekeeping mode only }\end{array}$ \\
STANDBY & Powered on, TEC on \\
LOW-RES & TEC on, CCD active, $64 \times 64$ image size, $4 \times 4$ binning \\
MED-RES & $\begin{array}{l}\text { TEC on, CCD active, } 128 \times 128 \text { image size, } 2 \times 2 \\
\text { binning }(\text { default for FAI-VIS with } 500 \text { ms exposure, }\end{array}$ \\
& $\begin{array}{l}30 \mathrm{~s} \text { cycle }) \\
\text { TEC on, CCD active, } 256 \times 256 \text { image size, } 1 \times 1\end{array}$ \\
HIGH-RES & $\begin{array}{l}\text { binning }(\text { default for FAI-NIR with } 100 \mathrm{~ms} \text { exposure, } \\
1.0 \mathrm{~s} \text { cycle })\end{array}$ \\
\hline
\end{tabular}

Table 3 Observations with default operating parameters

\begin{tabular}{lll}
\hline Parameter & VIS $(630 \mathrm{~nm})$ & NIR \\
\hline $\begin{array}{l}\text { Pixel array } \\
\begin{array}{l}\text { Projected pixel size } \\
\text { (from apogee) }\end{array}\end{array}$ & $128 \times 128$ & $256 \times 256$ \\
$\begin{array}{l}\text { Projected pixel size } \\
\text { (from perigee) }\end{array}$ & $0.4 \mathrm{~km}$ & $2.5 \mathrm{~km}$ \\
$\begin{array}{l}\text { Exposure time } \\
\text { Exposure interval }\end{array}$ & 0.5 second & $0.4 \mathrm{~km}$ \\
Sensitivity (SNR $>3)$ & 30 seconds & 0.1 second \\
& 200 rayleighs & $\begin{array}{l}100 \text { rayleighs } \\
(557.7 \text { nm equiv.) }\end{array}$ \\
\hline
\end{tabular}


Fig. 3 Spatial auroral coverage by the NIR camera from apogee at $1500 \mathrm{~km}$
Fig. 4 Photon transfer curve for the NIR CCD
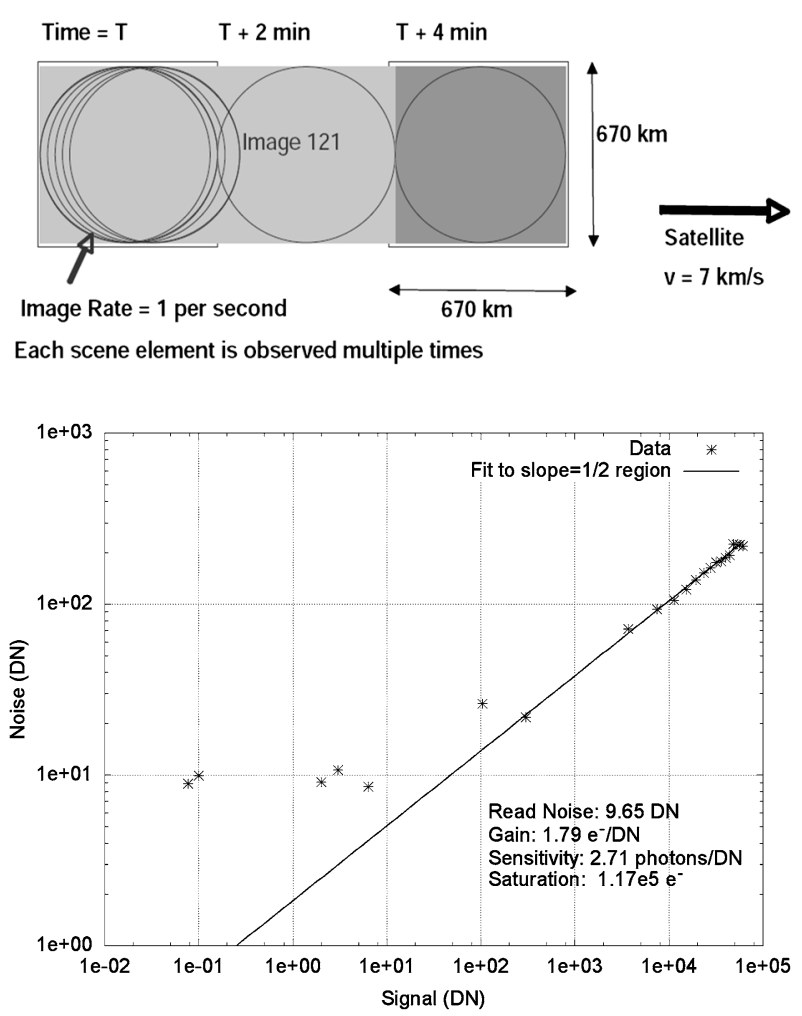

camera and subtends an angle of about $0.1^{\circ}$; this forms the basis for the given projected pixel size. Satellite motion during the exposure can of course degrade the spatial resolution. The effect is minimal when images are taken from apogee, even for a 0.5 second exposure in the case of the VIS camera. For the occasional perigee viewing, the satellite can be made to slew at the appropriate rate to compensate for the satellite motion; otherwise significant smearing can occur for the longer exposure times used by the VIS camera.

Shown in Fig. 3 is the projected field of view from apogee of the NIR camera. It is obvious that there is significant overlap between images that allows the same location in the aurora to be observed many times (up to about 120 depending on the location) thus permitting a complete record of both spatial and temporal variations over a period of 2 minutes. This provides a promising new capability to investigate small-scale auroral dynamics from space.

The sensitivity of the cameras is adequate to provide good images at sub-auroral, airglow intensity levels. Rather than rely on calculations for the responsivity of the cameras, absolute calibration measurements were made at the University of New Hampshire (UNH) using a calibrated integrating sphere as the source (Sadler 2014). The numerical values are given in Table 1. In addition to calibration, a number of measurements were made to provide the following: dark current for each pixel as a function of temperature; uniformity correction; distortion correction; baffle attenuation; and field of view. The photon transfer curve for each camera was also based on the laboratory measurements. Figure 4 shows the photon transfer curve for the NIR camera derived from images of the uniform light source at UNH. Image saturation was not reached during testing, causing the top end of the curve to be slightly cutoff. Fixed pattern noise was eliminated by subtracting two frames, leaving only shot noise. 
The operation schedule of the cameras on a daily basis is arrived at in consultation with members of the eight instrument teams as well as the satellite operations personnel. The FAI instrument has been scheduled to operate every 1-2 days for about 20 minutes, and during the first year of operation has collected more than 100,000 images. Satellite telemetry is routed between the mission operation center near Calgary, Canada and the Swedish Space Corporation facility in Kiruna, Sweden. S-band receiving sites have been utilized at O'Higgins in Antarctica, Inuvik in northern Canada, and Kiruna. Processing of FAI data has taken place at the University of Calgary. All images (in raw and png formats) are accessible to the public via the Canadian Space Science Data Portal at the University of Alberta (http://www.cssdp.ca). In addition, each sequence of images can be viewed as an mp4 video that also shows the source location of each image. Further analysis of images can be carried out using a purpose-developed set of IDL software tools called FAItools that can be distributed to potential users.

\section{Available Science Investigations}

An important feature of the Cassiope satellite for optimizing the science return is its attitude control system, which has four primary modes that are of value to FAI. The default mode is nadir viewing in which images are taken over a large latitude range during nighttime periods. At times there is a need for the cameras to utilize a second pointing mode that allows for pointing at a target fixed to the Earth such as an auroral observatory or rocket range, for example. The third mode is limb viewing when altitude profiles are of interest. The fourth mode is inertial pointing at a star field that is used to check camera coalignment, field of view, and focus. A summary of the science targets and the operational modes is given in Table 4. The FAI has many applications to a variety of auroral and airglow phenomena. In the polar cap there are transpolar arcs and enhanced airglow patches that drift from the dayside to the nightside over the polar region. In the main auroral oval there often exist multiple arcs that are not well understood. These are in addition to various dynamic substorm phenomena including the definition and observation of the onset. At lower latitudes there exist detached arcs that appear to be a signature of electrons that enter the magnetosphere on the dayside and drift around the Earth towards the evening sector.

Figure 5 shows one example of an auroral observation in the nadir mode taken with the NIR camera on December 8, 2013 at 04:40:38 UT. The satellite was at an altitude of $1400 \mathrm{~km}$ over northern Canada, near $62^{\circ}$ latitude. The exposure was $0.1 \mathrm{~s}$ and the repetition rate was one image per second. A second example is shown in Fig. 6 for November 9, 2013 at 04:45:49 UT. Cassiope was near $68^{\circ}$ latitude on the northeastern region of Siberia,

Table 4 FAI science targets

\begin{tabular}{|c|c|c|c|}
\hline Viewing Mode & Polar Cap & Auroral Oval & Mid/Low Latitudes \\
\hline \multirow[t]{3}{*}{ Nadir } & Polar arcs & Substorms & Detached arcs \\
\hline & Polar patches & Vortices & SAR arcs \\
\hline & Polar airglow & Multiple Arcs & \\
\hline Limb & Emission altitude profile & Emission altitude profile & Night airglow \\
\hline Earth Target & $\begin{array}{l}\text { Aurora and airglow } \\
\text { above station }\end{array}$ & $\begin{array}{l}\text { Aurora and airglow } \\
\text { above station }\end{array}$ & \\
\hline Inertial & Reference star field & Reference star field & Reference star field \\
\hline
\end{tabular}


Fig. 5 An auroral image obtained by the NIR camera
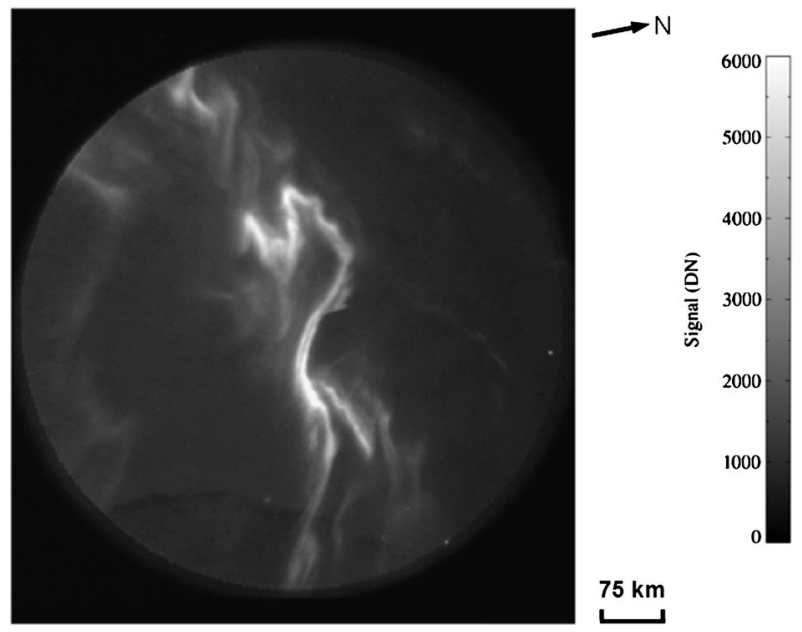

$75 \mathrm{~km}$

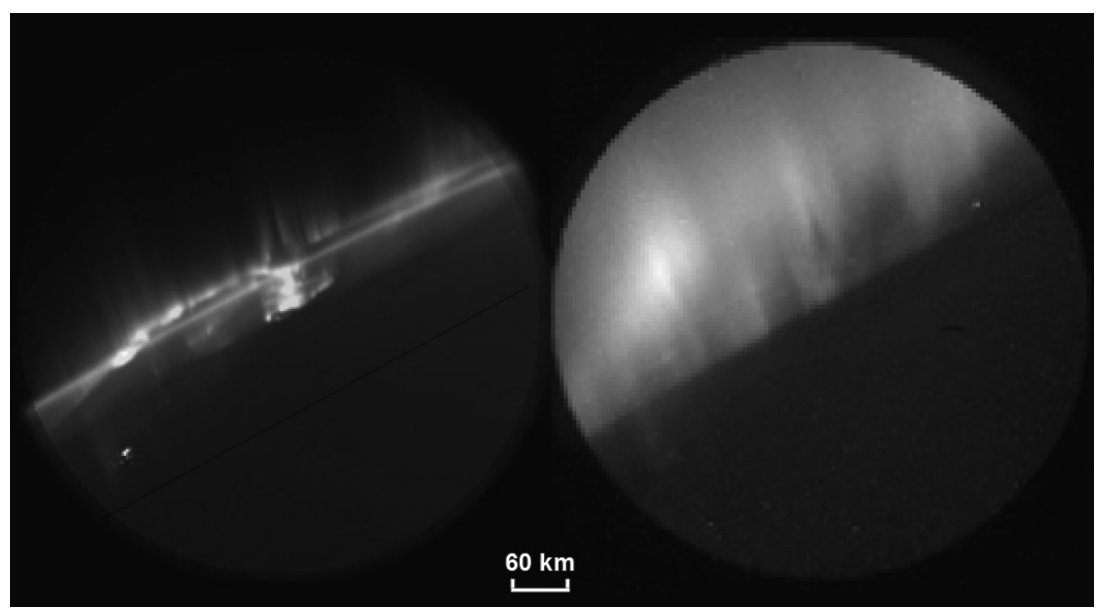

Fig. 6 Images taken of the limb using the NIR (left) and VIS (right) cameras. Note that the VIS image brightness has been scaled up by 4.4 times for clarity

looking eastward towards the limb. On the left, the aurora at the limb is clearly visible in the $650-1100 \mathrm{~nm}$ range. On the right is shown the $630 \mathrm{~nm}$ emission altitude projection above the limb, taken one second after the image on the left.

\section{Summary and Conclusion}

The Fast Auroral Imager (FAI) is part of the e-POP instrument payload on the Canadian Cassiope small satellite. The major scientific objectives of e-POP are to investigate plasma outflow from the polar regions and the related processes of microscale ion acceleration, wave-particle interaction, and auroral excitation, the 3D ionospheric irregularities using both active and passive radio techniques, and the escape of neutral particles from high latitudes caused by temperature enhancements as well as by non-thermal processes. The Fast Auroral 
Imager was developed to meet the optical imaging objectives of high temporal and spatial resolution imaging of mesoscale optical aurora from space in the mission.

Since the launch of the Cassiope satellite on September 29, 2013, emphasis has been placed on learning about the FAI instrument, its capabilities and limitations. For example, as expected, the nadir viewing mode has been the most useful especially for comparing with data from the in situ experiments. The quantity of data collected has been less than hoped for, and there are several reasons for this. The baffle was constrained in length by the satellite design and thus could not adequately eliminate sunlight. This meant that the FAI entrance had to be in shadow for successful operation. Another unexpected impact was the unavailability of the Ka-band telemetry downlink system that was expected to transmit about 10 times more data than has been realized. Finally, the satellite's radiation environment has caused a significant increase in the noise level of the CCDs. The NIR camera will last beyond one year but the VIS camera's performance deteriorated to the point that it was shut off in June 2014.

In conclusion, it is clear that the instrument provides an opportunity to significantly advance our understanding of auroral phenomena particularly in the mesoscale. In addition, it provides essential context images for the other observations on e-POP.

Acknowledgement Funding in Canada for this project has been provided by the Canadian Space Agency and the Natural Sciences and Engineering Research Council. Marc Lessard has received support from NASA for the characterization and calibration of the instrument.

Open Access This article is distributed under the terms of the Creative Commons Attribution License which permits any use, distribution, and reproduction in any medium, provided the original author(s) and the source are credited.

\section{References}

C.D. Anger, T. Fancott, J.M. McNally, H.S. Kerr, The ISIS-2 auroral scanning photometer. Appl. Opt. 17531766 (1973)

C.D. Anger, S.K. Babey, A.L. Broadfoot, R.G. Brown, L.L. Cogger, R. Gattinger, J.W. Haslett, R.A. King, D.J. McEwen, J.S. Murphree, E.H. Richardson, B.R. Sandel, K. Smith, A.V. Jones, An ultraviolet auroral imager for the Viking spacecraft. Geophys. Res. Lett. 14, 387-390 (1987)

J.L. Burch, IMAGE mission overview. Space Sci. Rev., IMAGE Special Issue 91, 1-14 (2000)

L.L. Cogger, E.F. Donovan, D.J. Knudsen, J.S. Murphree, T.S. Trondsen, Auroral imaging in Canada: Past, present and future. Can. Aeronaut. Space J. 48, 107-113 (2002)

L.A. Frank, J.D. Craven, Imaging results from Dynamics Explorer 1. Rev. Geophys. 26, 249-283 (1981)

G.A. Germany, J.F. Spann, G.K. Parks, M.J. Brittnacher, R. Elsen, L. Chen, D. Lummserzheim, M.H. Rees, Auroral observations from the POLAR Ultraviolet Imager (UVI), in Geospace Mass and Energy Flow: Results from the International Solar-Terrestrial Physics Program. Geophysical Monograph, vol. 104 (American Geophysical Union, Washington, 1998)

J.S. Murphree, M.L. Johnson, L.L. Cogger, D.J. Hearn, Freja UV Imager observations of spatially periodic auroral distortions. Geophys. Res. Lett. 21, 1887-1890 (1994)

E.B. Sadler, A low-level-light calibration facility and auroral precipitation driven density enhancement in the cusp. Ph.D. Thesis, University of New Hampshire (2014)

G. Walker et al., The MOST asteroseismology mission: ultraprecise photometry from space. Publ. Astron. Soc. Pac. 115, 1023-1035 (2003)

A.W. Yau, H.G. James, W. Liu, The Canadian Enhanced Polar Outflow Probe (e-POP) mission in ILWS. Adv. Space Res. 38, 1870-1877 (2006) 